\title{
Correction to: c-Jun Amino-Terminal Kinase is Involved in Valproic Acid-Mediated Neuronal Differentiation of Mouse Embryonic NSCs and Neurite Outgrowth of NSC-Derived Neurons
}

\author{
Lu Lu ${ }^{1} \cdot$ Hengxing Zhou ${ }^{1} \cdot$ Bin Pan ${ }^{1} \cdot$ Xueying $\mathrm{Li}^{2} \cdot$ Zheng Fu$^{2} \cdot$ Jun Liu ${ }^{1} \cdot$ Zhongju Shi $^{1} \cdot$ Tianci Chu $^{3} \cdot$ Zhijian Wei $^{1}$. \\ Guangzhi Ning ${ }^{1} \cdot$ Shiqing Feng ${ }^{1}$
}

Published online: 20 August 2019

(c) Springer Science+Business Media, LLC, part of Springer Nature 2019

\section{Correction to: \\ Neurochemical Research (2017) 42(4):1254-1266 \\ https://doi.org/10.1007/s11064-016-2167-7}

In the original version of this article, unfortunately, the images in Fig. 4 and 7 are mixed. The correct version of the Figs. 4 and 7 is given below.

The original article can be found online at https://doi.org/10.1007/ s11064-016-2167-7.

Shiqing Feng

fshiqing1967@ hotmail.com

1 Department of Orthopaedics, Tianjin Medical University General Hospital, No. 154 Anshan Road, Heping District, Tianjin 300052, People's Republic of China

2 Key Laboratory of Immuno Microenvironment and Disease of the Educational Ministry of China, Department of Immunology, Tianjin Medical University, No. 22 Qixiangtai Road, Heping District, Tianjin 300070 , People's Republic of China

3 Kosair Children's Hospital Research Institute at the Department of Pediatrics, University of Louisville School of Medicine, Louisville, KY 40202, USA 
Fig. 4 Mouse embryonic NSCs were treated with $1 \mathrm{mM} \mathrm{VPA}$ for up to 7 days and immunostained with anti- $\beta$-tubulinIII, anti-MAP-2, anti-CNPase, and anti-GFAP. Scale bar $100 \mu \mathrm{m}$

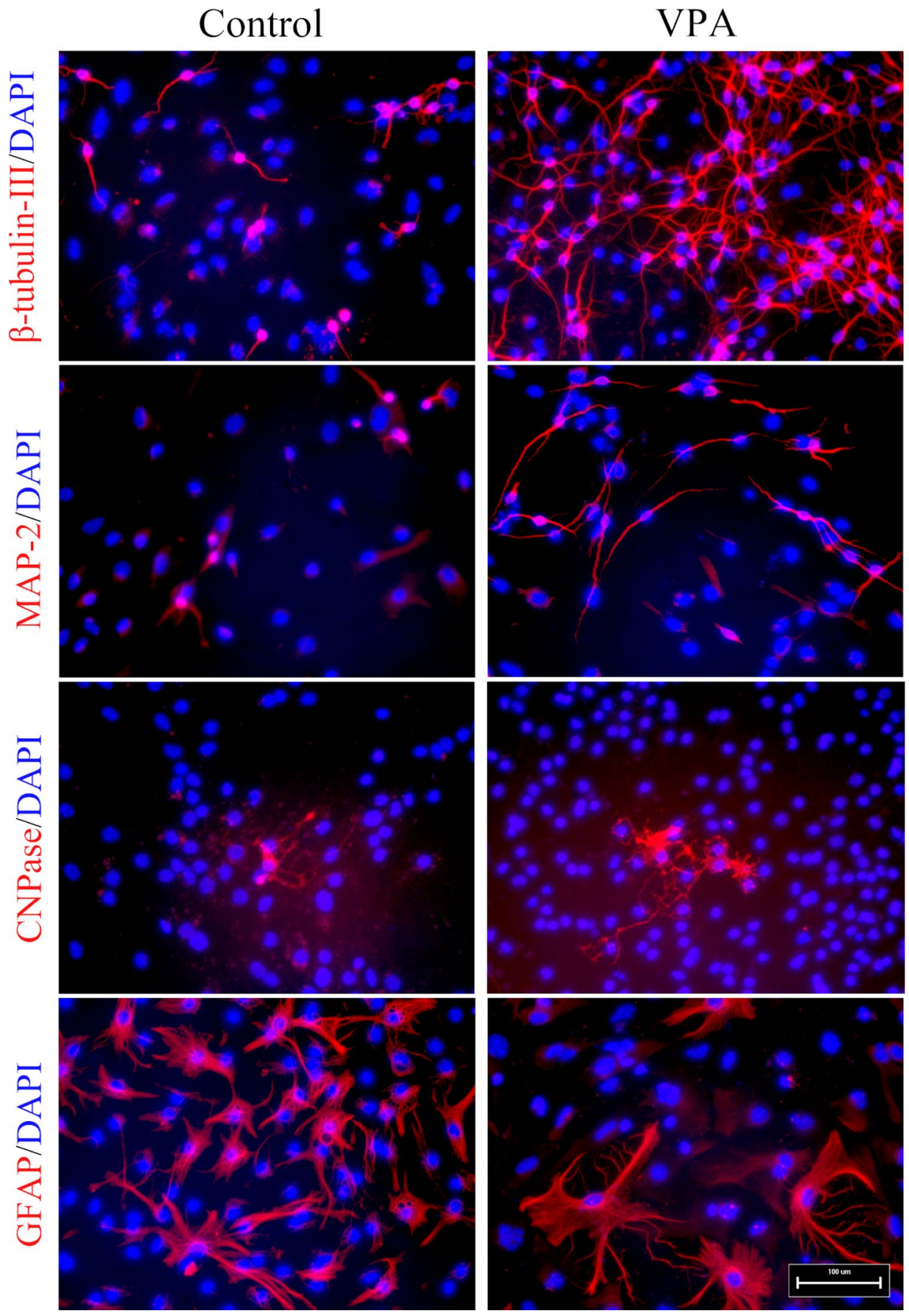




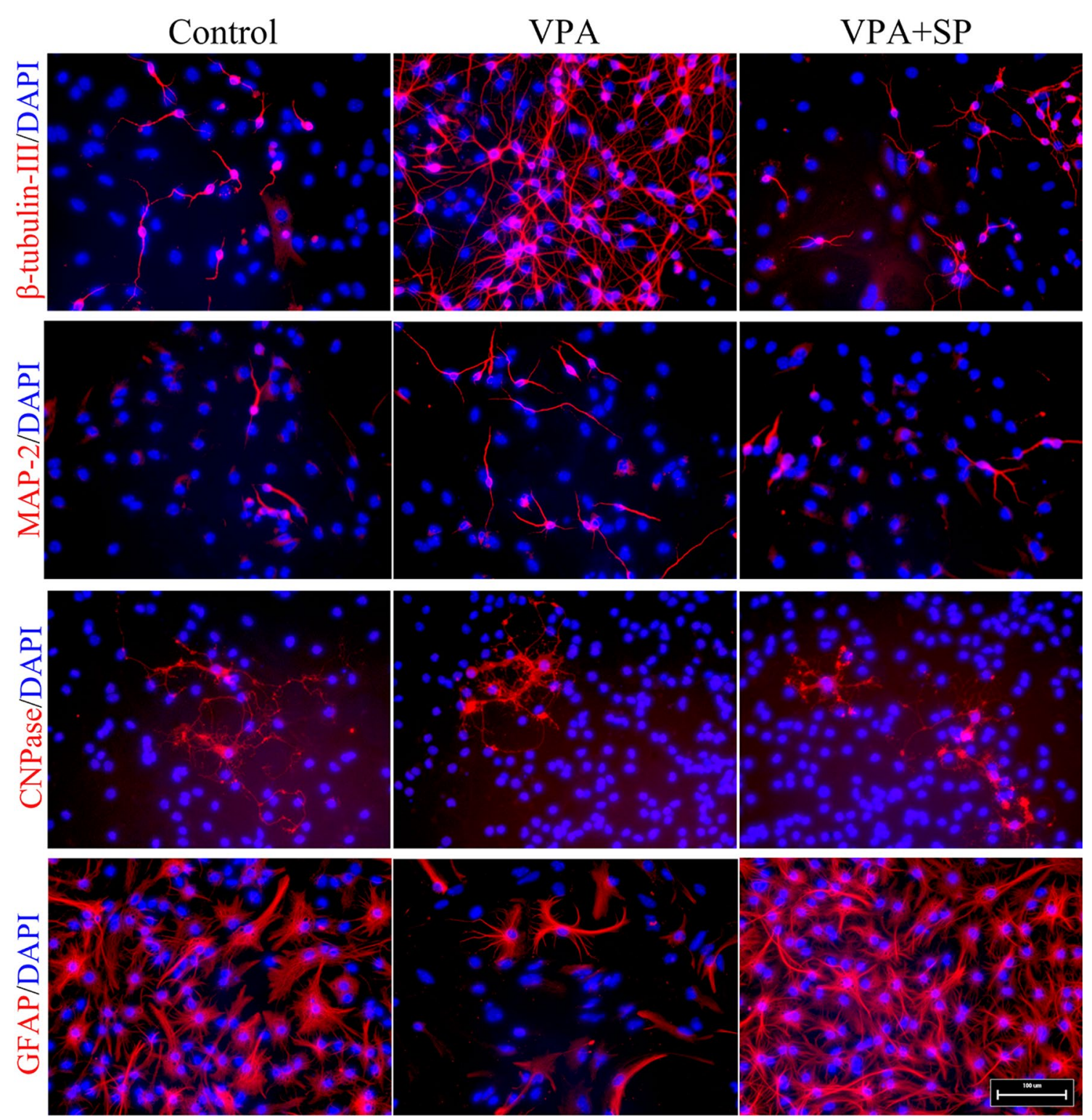

Fig. 7 Mouse embryonic NSCs were treated with $1 \mathrm{mM}$ VPA for up to 7 days in the absence or presence of $10 \mu \mathrm{M}$ SP600125 and immunostained with anti- $\beta$-tubulin-III, anti-MAP-2, anti-CNPase, and anti-GFAP. Scale bar $100 \mu \mathrm{m}$

Publisher's Note Springer Nature remains neutral with regard to jurisdictional claims in published maps and institutional affiliations. 